\title{
Photon path length distribution in random media from spectral speckle intensity correlations
}

\author{
L.F. Rojas ${ }^{1}$, M. Bina ${ }^{2}$, G. Cerchiari ${ }^{3}$, M.A. Escobedo-Sánchez ${ }^{1}$, F. Ferri², \\ and F. Scheffold ${ }^{4, a}$ \\ 1 Departamento de Física, Cinvestav-IPN, Av. Instituto Politécnico Nacional 2508, \\ 07360 México D.F., México \\ 2 Dipartimento di Fisica e Matematica, Università degli Studi dell'Insubria, via Valleggio \\ 11, 22100 Como, Italy \\ 3 Dipartimento di Fisica, Università degli studi di Milano, via Celoria 16, 20133 Milano, \\ Italy \\ 4 Department of Physics and Fribourg Center for Nanomaterials, University of Fribourg, \\ 1700 Fribourg, Switzerland
}

Received 29 August 2011 / Received in final form 4 October 2011

Published online 7 December 2011

\begin{abstract}
We show that the spectral speckle intensity correlation (SSIC) technique can be profitably exploited to recover the path length distribution of photons scattered in a random turbid medium. We applied SSIC to the study of Teflon slabs of different thicknesses and were able to recover, via the use of the photon diffusion approximation theory, the characteristic transport mean free path $\ell^{*}$ and absorption length $s_{a}$ of the medium. These results were compared and validated by means of complementary measurements performed on the same samples with standard pulsed laser time of flight techniques.
\end{abstract}

\section{Introduction}

The propagation of waves in random media depends on refractive index fluctuations and structural composition of the scattering medium [1]. Understanding the optical properties of dense disordered materials is not only a problem of fundamental interest but also a key issue in the development of noninvasive probing tools, e.g. near-IR light imaging in biological tissue and characterization of the visco-elastic properties of many gel-like materials of industrial interest [2]. In the absence of long-range structural order, light is spread out diffusively and its transport can be described as a diffusion process with a characteristic step set by the transport mean free path, the typical distance after which a photon loses memory on its initial direction. Biological tissue belongs to an important class of random media where the transport of light can be considered diffusive.

\footnotetext{
a e-mail: frank.scheffold@unifr.ch
} 
The interference of multiply scattered coherent partial waves in a disordered medium produces aleatory fluctuations of the measured intensity, which is known as the speckle phenomenon [3]. The statistical properties of this speckle pattern carry information on the scattering properties of the disordered medium and on the coherence properties of the illuminating source [4,5]. For example, the second-order intensity cross-correlation of the light multiply scattered by a disordered medium at two different laser frequencies can be studied as a function of the frequency difference, and it is known to be related to the temporal response of the medium $[6,7]$. However, this time response can be obtained from second-order intensity correlations only when information is used a priori, e.g. by assuming a form of the temporal response from the photon diffusion model, and fitting unknown parameters [8-10]. On the other hand, Blount and Klauder [11] established that third-order correlations are sufficient to recover the shape of the multiple scattered intensity pulse when the medium is excited with a short laser pulse. More recently, Webster et al. [12,13] have introduced the use of third-order spectral speckle intensity correlations (SSIC) as a means to determine the temporal response of random media. In Webster's model, no assumptions on the form of the temporal response are necessary by using third-order spectral intensity correlations. In this model, any multiple scattering disordered medium can be studied, provided that the scattered fields obey circular complex Gaussian statistics [4].

In this work, we present a review and an experimental test of the spectral SSIC technique by using slabs of Teflon of different thickness $L$ as model systems. Following Webster's approach $[12,13]$, we were able to determine the transport mean free path $\ell^{*}$ and the absorption length $s_{a}$ of Teflon, which, to our knowledge, has never been characterized with this method. We also tested the performance and the reliability of the technique by complementary measurements of the temporal response of the same Teflon samples with standard photon time of flight techniques [8-10], based on the use of short laser pulses and time resolved detection of scattered light. The experimental results are compared with theoretical predictions based on the photon diffusion approximation (DA) theory, which can be applied in this case because the requirements of weak scattering $\left(\ell^{*}>\lambda\right.$, being $\lambda$ the wavelength of radiation), diffusive regime $\left(\ell^{*} \ll s_{a}, \ell^{*} \ll L\right)$, and absence of long- and infinite-range correlations effects [14], are all fulfilled for our systems.

The paper is organized as follows: in Section 2, the model for the scattered field from random media is presented and the required conditions to apply Gaussian statistics are established. Then, the spectral speckle intensity correlation method is reviewed by recalling the properties of the field and intensity correlations, introducing the concept of the intensity bispectrum, and describing how it can be exploited to reconstruct the temporal response of the medium via second- and thirdorder measured intensity correlations. This section finalizes with a review of the notions behind the diffusion approximation for light transport in random media. Section 3 details the experimental setup to measure the frequency-dependent speckle patterns in transmission geometry and reports about first- and second-order statistical properties of the measured intensity speckle patterns. Then a description of the protocol used to construct third-order correlation functions and the corresponding bispectrum is also included. The section ends describing the setup implemented for the time of flight measurements, that were used for validating the results obtained with the SSIC technique. In Section 4, the experimental results derived from the two techniques are compared and interpreted in terms of the diffusion approximation theory that describes light transport in turbid random media. Finally, Section 5 is devoted to our conclusions in view of the results obtained in this work. 


\section{Theoretical background}

\subsection{Scattered field from random media}

When a coherent beam of light is scattered by a random medium, it produces fluctuations of the measured intensity due to interference of many scattered partial waves, this phenomenon being known as speckle pattern [3,4]. The total field in a small area of the speckle pattern can be expressed as the superposition of many scattered partial waves

$$
E_{0}(\nu)=\sum_{k=1}^{N} A_{k} \exp \left[-i \phi_{k}(\nu)\right]
$$

where $A_{k}$ is the $k_{\text {th }}$ aleatory field magnitude, considered to be frequency independent, and $\phi_{k}(\nu)$ is the $k_{\text {th }}$ random phase of a partial wave. Several assumptions over the statistical properties of the scattered partial waves are implicit in the random phasor sum model of Goodman [3,4]: i) the aleatory field magnitudes $A_{k}$ are statistically independent and identically distributed, ii) the random field phases $\phi_{k}(\nu)$ are statistically independent and uniformly distributed over the interval $-\pi$ to $\pi$ and, iii) the aleatory magnitude $A_{k}$ and the random phase $\phi_{k}(\nu)$ of each partial wave are both statistically independent. These assumptions are equivalent to specifying that the random paths of each partial wave scattered through a disordered medium are all statistically equivalent and independent. This allows to model $\phi_{k}(\nu)$ as an accumulated phase after traversing the $k_{\text {th }}$ path whose random time of flight is $t_{k}$, thus resulting in $\phi_{k}(\nu)=2 \pi \nu t_{k}$. Under the assumptions i)-iii), $E_{0}(\nu)$ is the sum of a large number of independent terms and, because of the central limit theorem, is described by a circular complex Gaussian statistics $[3,4]$.

\subsection{Spectral correlations of the scattered field and intensity}

Genack has derived an expression for the second-order field correlation between two scattering fields at different frequencies $[8,9]$

$$
\left\langle E_{0}(\nu+\Delta \nu) E_{0}^{*}(\nu)\right\rangle=\langle I\rangle P(\Delta \nu)
$$

where $P(\Delta \nu)$ is identified as the Fourier transform of the times of flight distribution $p(t)$ and it is represented by

$$
P(\Delta \nu)=\int_{-\infty}^{\infty} \mathrm{d} t p(t) \exp (-2 \pi i \Delta \nu t)
$$

Equation (3) shows that, if the times of flight distribution is assumed to have an approximated spread $\Delta t$, the scattered field $E_{0}(\nu)$ will become uncorrelated with $E_{0}(\nu+\Delta \nu)$ when the change in frequency $\Delta \nu$ satisfies the condition $\Delta \nu \Delta t \approx 1$. This condition takes place when the difference between the accumulated phases of the two scattered fields can be assumed to be approximately random over $2 \pi$.

For electromagnetic waves scattered from a turbid random medium, the observable is the second-order intensity correlation $\langle I(\nu+\Delta \nu) I(\nu)\rangle$. Since the field statistics are 
circular complex Gaussian, the Gaussian moment theorem [15] can be applied together with Eq. (2) to give

$$
\langle I(\nu+\Delta \nu) I(\nu)\rangle=\langle I\rangle^{2}+\langle I\rangle^{2}|P(\Delta \nu)|^{2} .
$$

On the other hand, it is mathematically more convenient to define a normalized intensity $\tilde{I}=(I-\langle I\rangle) /\langle I\rangle$, allowing to rewrite the second-order correlation as

$$
\langle\tilde{I}(\nu+\Delta \nu) \tilde{I}(\nu)\rangle=|P(\Delta \nu)|^{2} .
$$

The second-order intensity correlation contains information only about the Fourier magnitude of $p(t)$. Since the Fourier phase information is lost, it is not possible to reconstruct $p(t)$ from measurements of second-order intensity correlations without having a priori information. Nevertheless, it has been previously shown that the thirdorder intensity correlations do contain sufficient information about the Fourier phase of $p(t)[12,13]$. This enables the reconstruction of the probability density function of the times of flight from intensity based measurements. The third-order intensity correlation can be evaluated by the Gaussian moment theorem [15], Eq. (2) and the normalized intensity $\tilde{I}$ to give

$$
\begin{aligned}
& \left\langle\tilde{I}(\nu) \tilde{I}\left(\nu+\Delta \nu_{1}\right) \tilde{I}\left(\nu+\Delta \nu_{1}+\Delta \nu_{2}\right)\right\rangle= \\
& 2 \operatorname{Re}\left\{P\left(\Delta \nu_{1}\right) P\left(\Delta \nu_{2}\right) P^{*}\left(\Delta \nu_{1}+\Delta \nu_{2}\right)\right\} .
\end{aligned}
$$

Webster et al. $[12,13]$ identified that the third-order intensity correlation in Eq. (6) is equal to twice the real part of the bispectrum of $p(t)$, hence, the use of bispectral techniques is possible in the reconstruction of $p(t)$.

As pointed out in Ref. [12,13], second-order correlations can be related to the Fourier magnitude of $p(t)$ and the phase information can be obtained from the thirdorder correlations, which could be used together to reconstruct the temporal response from turbid random media. To establish the reconstruction method, first consider the third-order temporal correlation of a real function $f(t)$ as

$$
g^{(3)}\left(\tau_{1}, \tau_{2}\right)=\int_{-\infty}^{\infty} \mathrm{d} t f(t) f\left(t+\tau_{1}\right) f\left(t+\tau_{2}\right),
$$

and the Fourier transform of the third-order temporal correlation as

$$
G^{(3)}\left(\nu_{1}, \nu_{2}\right)=\int_{-\infty}^{\infty} \mathrm{d} \tau_{1} \int_{-\infty}^{\infty} \mathrm{d} \tau_{2} g^{(3)}\left(\tau_{1}, \tau_{2}\right) \exp \left[-2 \pi i\left(\nu_{1} \tau_{1}+\nu_{2} \tau_{2}\right)\right],
$$

which can be interpreted as the bispectrum of $f(t)$ and also can be expressed in terms of its Fourier transform $F(\nu)[12,13]$

$$
f(t)=\int_{-\infty}^{\infty} \mathrm{d} \nu F(\nu) \exp (2 \pi i \nu t)
$$

This allows us to write the bispectrum of $f(t)$ by the product

$$
G^{(3)}\left(\nu_{1}, \nu_{2}\right)=F\left(\nu_{1}\right) F\left(\nu_{2}\right) F\left(-\nu_{1}-\nu_{2}\right) .
$$

Since $f(t)$ is a real function, then the identity $F\left(-\nu_{1}-\nu_{2}\right)=F^{*}\left(\nu_{1}+\nu_{2}\right)$ can be used in Eq. (10), giving the result

$$
G^{(3)}\left(\nu_{1}, \nu_{2}\right)=F\left(\nu_{1}\right) F\left(\nu_{2}\right) F^{*}\left(\nu_{1}+\nu_{2}\right) .
$$


The Fourier transform of $f(t)$ can be expressed in terms of its magnitude and phase as $F(\nu)=A(\nu) \exp [i \phi(\nu)]$, and the bispectrum in terms of its magnitude and phase as $G^{(3)}\left(\nu_{1}, \nu_{2}\right)=B\left(\nu_{1}, \nu_{2}\right) \exp \left[i \psi\left(\nu_{1}, \nu_{2}\right)\right]$. Then, the relationship between the Fourier phase and the bispectral phase of $f(t)$ becomes

$$
\psi\left(\nu_{1}, \nu_{2}\right)=\phi\left(\nu_{1}\right)+\phi\left(\nu_{2}\right)-\phi\left(\nu_{1}+\nu_{2}\right) .
$$

Equation (12) establishes that the bispectral phase is a linear combination of Fourier phases. By writing the Fourier and bispectral phases in discrete notation, where $\phi_{k}=$ $\phi(k \delta \nu)$ is the $k_{\mathrm{th}}$ Fourier phase, $\psi_{i, j}=\psi(i \delta \nu, j \delta \nu)$ is the $(i, j)_{\mathrm{th}}$ component of the bispectral phase, and $\delta \nu$ is the sample frequency increment, the Fourier phase can be reconstructed using Eq. (12)

$$
\phi_{k}=\frac{1}{k-1} \sum_{i=1}^{k-1} \phi_{i}+\phi_{k-i}-\psi_{i, k-i}, k=2, \ldots, N,
$$

where $\phi_{0}=0$, and $\phi_{1}=\phi(\delta \nu)$ is arbitrary, and $N$ is the number of samples.

By comparing Eq. (11) and Eq. (6), the third-order speckle intensity frequency correlation from a disordered medium is equal to twice the real part of the bispectrum of the temporal response $p(t)[16]$. Defining the normalized intensities of $\tilde{I}_{1}=\tilde{I}\left(\nu_{0}\right)$, $\tilde{I}_{2}=\tilde{I}\left(\nu_{0}+\Delta \nu_{1}\right)$ and $\tilde{I}_{3}=\tilde{I}\left(\nu_{0}+\Delta \nu_{1}+\Delta \nu_{2}\right)$, the bispectral phase is obtained from second- and third-order correlations using

$$
\psi\left(\Delta \nu_{1}, \Delta \nu_{2}\right)= \pm \cos ^{-1}\left[\frac{\left\langle\tilde{I}_{1} \tilde{I}_{2} \tilde{I}_{3}\right\rangle}{2\left(\left\langle\tilde{I}_{1} \tilde{I}_{2}\right\rangle\left\langle\tilde{I}_{2} \tilde{I}_{3}\right\rangle\left\langle\tilde{I}_{3} \tilde{I}_{1}\right\rangle\right)^{1 / 2}}\right] .
$$

The bispectral phase from Eq. (14) is then used in Eq. (13) to reconstruct the Fourier phase $[16,17]$. The full bispectral phase cannot be directly obtained from the speckle correlation measurements, but only the cosine of the bispectral phase. The main consequence of this is a sign ambiguity in the reconstructed Fourier phase, leading to time reversal indeterminacy in the temporal response. A more severe limitation occurs if the Fourier phase is not monotonic and its derivative changes sign over a reconstruction interval $\delta \nu$. However, the form of the temporal response from the turbid disordered medium used for this study has a monotonic Fourier phase, which is expected to be true for similar random media of practical interest.

Summarizing, in order to reconstruct the temporal response $p(t)$, the entire (complex) Fourier transform $P(\Delta \nu)=A(\Delta \nu) \exp [i \phi(\Delta \nu)]$ is needed. The amplitude $A(\Delta \nu)$ is found by taking the square root of the second-order correlation given in Eq. (5), while the phase $\phi(\Delta \nu)$ is recovered by using the bispectral techniques outlined above, throughout Eq. (5) and Eq. (14). The desired temporal response is then reconstructed by applying a Discrete Fourier transform (DFT) $p(t)=\int_{-\infty}^{\infty} \mathrm{d} \Delta \nu A(\Delta \nu) \exp [i \phi(\Delta \nu)][12,13]$.

As a final comment, we notice that this procedure leads to the correct recovery of the temporal response $p(t)$, but aside for an arbitrary time shift. This is due to the fact that Eq. (14) is blind to any linear phase shift of the Fourier phase $\phi(\nu)$, as it can be easily shown by replacing $\phi(\nu)$ by $\phi(\nu)=\phi(\nu)+\alpha \nu$. Thus, the linear phase arbitrariness in the frequency domain implies a time-offset indetermination of the signal in the time domain.

\subsection{Photon diffusion approximation}

The transport of light in random media in the weak scattering regime, i.e. when the transport mean free path is large compared with the source wavelength, is well 
described by the photon diffusion equation $[18,19]$

$$
\frac{\partial}{\partial t} \Phi(\mathbf{r}, t)+\frac{v}{s_{a}} \Phi(\mathbf{r}, t)-\nabla \cdot[D \nabla \Phi(\mathbf{r}, t)]=v S_{0}(\mathbf{r}, t),
$$

where $\Phi(\mathbf{r}, t)$ is the angle-integrated photon flux density having units of (photon $\left.\mathrm{m}^{-2} \mathrm{~s}^{-1}\right), S_{0}(\mathbf{r}, t)$ represents an isotropic light source and $v$ is the effective transport velocity of the photons in the random medium. The diffusion coefficient $D$ has units of $\left(\mathrm{m}^{2} \mathrm{~s}^{-1}\right)$ and is given by

$$
D=\frac{v}{3\left[1 / s_{a}+1 / \ell^{*}\right]} \approx \frac{v \ell^{*}}{3},
$$

where $\ell^{*}$ is the transport mean free path and $s_{a}$ is the absorption length.

The right hand side of Eq. (16) is valid in the limit of $\ell^{*} \ll s_{a}$, which corresponds to conditions required for the strict validity of the diffusion approximation (DA). In what follows we will always consider the DA valid and set $D=v \ell^{*} / 3$.

The intensity measured by a photodetector $I_{\text {meas }}(t)$ is related to the photon current density $\mathbf{J}(\mathbf{r}, t)=(D / v) \nabla \Phi(\mathbf{r}, t)[19]$ by

$$
I_{\text {meas }}(t)=K \int_{A_{d}} d A \hat{\mathbf{n}} \cdot \mathbf{J}(\mathbf{r}, t),
$$

where $A_{d}$ is the photodetector area, $\hat{\mathbf{n}}$ is the photodetector surface normal vector and $K$ is a constant proportional to the photodetector numerical aperture and quantum efficiency. Under the diffusion approximation, the intensity temporal response of a random medium can be defined as the normalized Green's function of Eq. (15). Hence, with a source term of $S_{0}=\delta\left(\mathbf{r}^{\prime}, t\right)$, where $\mathbf{r}^{\prime}$ is the source location, Eq. (15) and (17) can be solved to obtain the intensity temporal response as

$$
p(t)=\frac{I_{\text {meas }}(t)}{\int_{-\infty}^{\infty} \mathrm{d} t I_{\text {meas }}(t)} .
$$

The function $p(t)$ represents the time of flight distributions of all the photons traveling through the random turbid medium.

For a statistically homogeneous slab of thickness $L$ of a scattering medium, it is possible to obtain a quasi-analytical solution to the diffusion equation of Eq. (15) in transmission geometry, and hence the temporal response or photon path length distribution $[8,9,20]$. This is achieved by solving Eq. (15) with the boundary conditions that the photon density is zero at the slab interfaces. The path length distribution is calculated at $L-\ell^{*}$ which is essentially the distribution of the transmitted light. Since the light is detected in the far field, it emerges from all points in the output face. Thus, integrating over the output face gives $[8,9]$

$$
\begin{aligned}
P_{\text {theo }}(s, L)= & \frac{\sqrt{3} \exp \left(-s / s_{a}\right)}{\left(4 \pi s \ell^{*}\right)^{1 / 2}} \sum_{n=-\infty}^{\infty}\left\{\exp \left[\frac{-3\left[(2 n-1) L-2 \ell^{*}\right]^{2}}{4 s \ell^{*}}\right]\right. \\
& \left.-\exp \left[\frac{-3[(2 n-1) L]^{2}}{4 s \ell^{*}}\right]\right\},
\end{aligned}
$$

where we have assumed that $s=v t$ and $D=v \ell^{*} / 3$. Only terms up to $n=3$ contribute significantly to any fit of path length distributions with Eq. (19) $[8,9]$. 


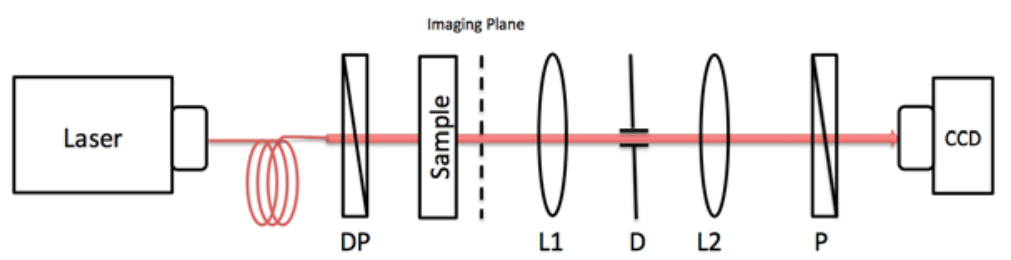

Fig. 1. Scheme of the experimental setup for the spectral speckle intensity correlations measurements. The laser light, emitted at $784.5 \mathrm{~nm}$, is coupled into an optical fiber ending with a collimator and then passed through a depolarizer $D P$ before being directed onto the sample. The imaging system consists of a unity magnification spatial filter in $4 f$ telescope configuration, which allows to control the speckle size at the plane in the CCD camera by removing the high frequency contributions in the Fourier space. The imaging systems is composed of lens $L 1, L 2$ with focal distance $f=75 \mathrm{~mm}$ and a diaphragm $D$ of diameter of about $5 \mathrm{~mm}$ located in the Fourier plane of the filter. A polarizer $P$ is placed in front of the CCD camera to analyze the light scattered by the sample in different polarization channels.

\section{Experimental setup}

\subsection{Spectral speckle intensity correlations measurements}

The experimental setup used to obtain the spectral speckle intensity correlations is shown in Fig. 1. It consists of a linearly polarized frequency tunable diode laser source (Toptica photonics, model DL $100 \mathrm{DFB}$ ) that operates at a single mode with a narrow line-width having a center wavelength of $\lambda=784.5 \mathrm{~nm}$. The center frequency can be linearly tuned over a range of $\approx 500 \mathrm{GHz}$ by changing the diode temperature. The range of frequencies used during the experiments was of $\approx 162 \mathrm{GHz}$. The radiation emitted by the laser is fed into an optical fiber, collimated by a lens coupled at its end face, and shined onto the front face of the scattering medium after passing through a Hanle depolarizer DP (Halle Nachfolger, Germany). All the optical elements were aligned along the optical axis. A small area of $\approx 2 \mathrm{~mm}^{2}$ on the back face of the scattering medium is imaged onto a CCD camera with $659 \times 493$ pixels of size $9.9 \mu \mathrm{m} \times$ $9.9 \mu \mathrm{m}$ (Prosilica GC655 of 12 bits dynamic range). The imaging system consists of a spatial filter in a $4 f$ telescope configuration of unity magnification. The spatial filter is formed by two identical achromatic lenses, $L 1$ and $L 2$, of focal length $f=75 \mathrm{~mm}$ and an adjustable iris aperture with a typical diameter of $D \approx 5 \mathrm{~mm}$, located at the Fourier focal plane. The diaphragm is used to control the numerical aperture of the collected light, which, in turn, determines the size of the speckles formed on the CCD camera. In our experiments, the size of the speckles on the camera was adjusted to be of about $3 \times 3$ pixels. A linear polarizer $P$ was placed in front of the CCD in order to select light scattered by the sample in single polarization channels. Exposure times of about $100 \mathrm{~ms}$ were used by the CCD camera to acquire a speckle image. The frequency scan is performed by applying a periodic triangular current wave signal to the laser controller in order to change the temperature of the diode thus obtaining a linear dependence of the central frequency in time. A typical value of $200 \mathrm{~s}$ is used as the period of the ramp, which allows to scan in frequency increments of $\approx 0.270 \mathrm{GHz}$. As frequency is changed, we acquire 600 images of the speckle pattern, each one corresponding to an individual frequency shift. In this configuration, we are able to scan around $162 \mathrm{GHz}$, enough to investigate the full decay of the intensity correlations in our random turbid media.

A typical measured speckle intensity pattern from a Teflon slab, thickness of $9.25 \mathrm{~mm}$, is shown in Fig. 2. The intensity histogram of this figure provides an estimate of the first-order speckle intensity statistics, shown in Fig. 3. The 


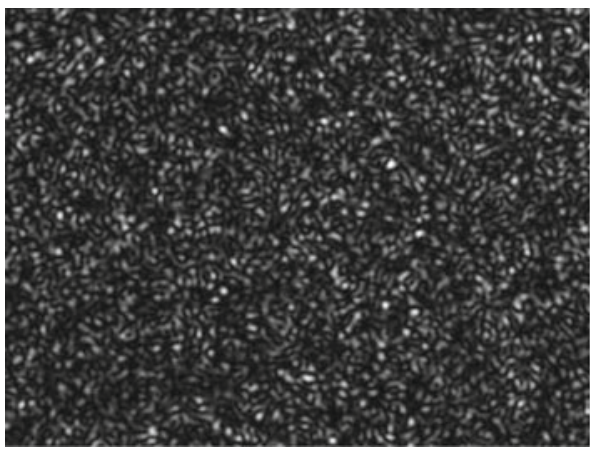

Fig. 2. Typical speckle pattern from a Teflon slab of thickness of $9.25 \mathrm{~mm}$ as obtained by the experimental setup shown in Fig. 1.

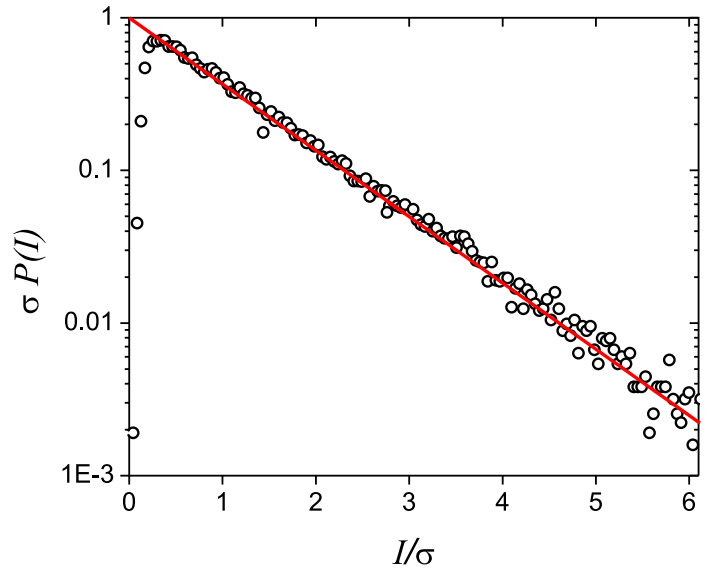

Fig. 3. Intensity histogram obtained from the speckle pattern shown in Fig. 2 (symbols). The negative exponential distribution expected for zero-mean circular complex Gaussian field statistics is shown as solid line.

histogram is closely modeled by a negative exponential probability distribution function, $\sigma P_{I}(I)=\exp (-I / \sigma)[3,4]$, where $\sigma$ is the standard deviation of the measured speckle pattern $\sigma /\langle I\rangle \simeq 0.87$. The latter value is somewhat smaller than the ideal ratio $\sigma /\langle I\rangle=1$ because of the detector finite pixel size, which in our case is only $\sim 1 / 3$ of the speckle size.

The second-order intensity correlation of Eq. (5) depends only on the frequency shift between measurements. Therefore, an average over each frequency combination pair that gives the same frequency shift is performed to form the spectral intensity second-order correlation data point for that particular frequency difference. Denoting the normalized intensity sample at each frequency increment $\tilde{I}_{i}=\tilde{I}(\nu+i \delta \nu)$ for $i=0,1, \ldots, N-1$, then the averaged second-order intensity correlation of Eq. (5) is determined by $[12,13]$

$$
\langle\tilde{I}(\nu) \tilde{I}(\nu+\Delta \nu)\rangle_{\text {meas }}=\frac{1}{N-i} \sum_{j=0}^{N-i-1}\left\langle\tilde{I}_{j} \tilde{I}_{j+i}\right\rangle,
$$

with $\Delta \nu=i \delta \nu$ and $i=0,1, \ldots, N-1$. The quantity $\left\langle\tilde{I}_{j} \tilde{I}_{j+i}\right\rangle$ represents the estimate of the second-order intensity correlation evaluated for frequencies $\nu+j \delta \nu$ and $\nu+$ $(j+i) \delta \nu$. The results for the spectral intensity second-order correlations are shown 


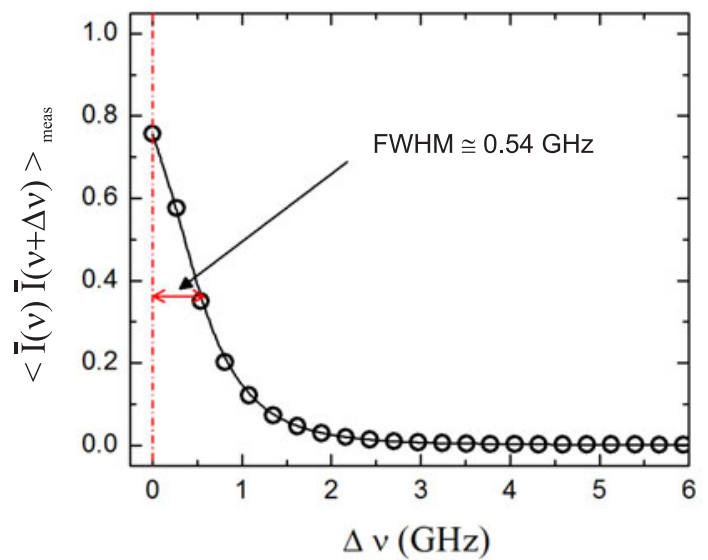

Fig. 4. Measured spectral intensity second-order correlation defined in Eq. (20) for a Teflon slab of thickness $L=9.25 \mathrm{~mm}$ in transmission geometry.

in Fig. 4. Notice that the amplitude of $\langle\tilde{I}(\nu) \tilde{I}(\nu+\Delta \nu)\rangle_{\text {meas }}=\left\langle\tilde{I}^{2}\right\rangle_{\text {meas }} \approx 0.76$ is consistent with the value estimated from the first order statistics, e.g. $\left\langle\tilde{I}^{2}\right\rangle \sim(\sigma /\langle I\rangle)^{2}$, and it is due, as in the case of Fig. (3), to finite detector size effects. However, this is only a scaling prefactor, which does not affect the circular Gaussian statistical properties of the speckle fields, and therefore, does not prevent the proper applicability of the technique.

To calculate the third-order intensity correlation of Eq. (6), an average is performed of each frequency triplet that gives the same frequency shifts for $\Delta \nu_{1}$ and $\Delta \nu_{2}$ to form each measured third-order correlation data point. Averaging all possible combinations gives the measured third-order intensity correlation $[12,13]$

$$
\begin{aligned}
\left\langle\tilde{I}(\nu) \tilde{I}\left(\nu+\Delta \nu_{1}\right) \tilde{I}\left(\nu+\Delta \nu_{1}+\Delta \nu_{2}\right)\right\rangle_{\text {meas }}= & \\
& \frac{1}{N-i-j} \sum_{k=0}^{N-1-i-j}\left\langle\tilde{I}_{k} \tilde{I}_{k+i} \tilde{I}_{k+i+j}\right\rangle,
\end{aligned}
$$

where $\Delta \nu_{1}=i \delta \nu, \Delta \nu_{2}=j \delta \nu$, and $i, j=0,1, \ldots, N-1$ with $i+j \leq N-1$. The quantity $\left\langle\tilde{I}_{k} \tilde{I}_{k+i} \tilde{I}_{k+i+j}\right\rangle$ represents the estimate of the third-order intensity correlation evaluated at the frequencies of $\nu+k \delta \nu, \nu+(k+i) \delta \nu$, and $\nu+(k+i+j) \delta \nu$.

\subsection{Time of flight measurements}

The accuracy and reliability of the SSIC technique in recovering the photon path length distribution was ascertained by complementary time resolved measurements carried out on the same samples with a ps-pulsed excitation and a time correlated single photon counting (TCSPC) technique. The setup used for this measurements is described in [22] and sketched in Fig. 5. A train of equally spaced and suitably attenuated laser pulses is used as a light probe and excite the random medium so that, for each pulse, there is only one (multiple) scattered photon reaching the detector. This photon is detected with a Single Photon Avalanche Detector (SPAD, Micro photon devices, model PDM50 sensitive area $\approx 50 \mu \mathrm{m}^{2}$ ) combined with a Time To Amplitude converter (TAC) and a Multichannel Analyzer (MCA) integrated in a single card (Becker\&Hickl, model: SPC 150) installed in the computer. The pulsed 


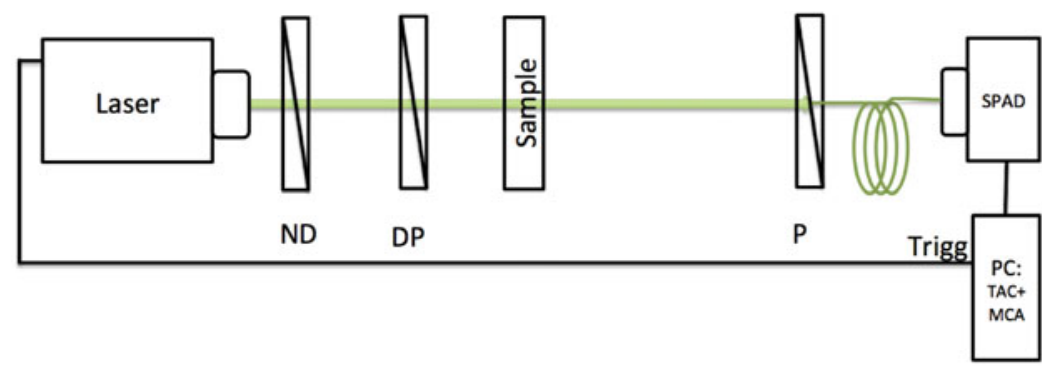

Fig. 5. Scheme of the experimental setup for the time of flight measurements. The laser pulse emitted at $532 \mathrm{~nm}$ was attenuated by a neutral density $(N D)$ filter, passed through a depolarizer $(D P)$, and hit the target. A polarizer $P$ was mounted after the sample to analyze the diffuse light in different polarization channels. The light was collected by bare optical fiber with a $800 \mu \mathrm{m}$ core diameter, and focussed via an optical microscope on the sensitive area of a Single Photon Avalanche Detector (SPAD). The path length distribution was reconstructed by using a Time To Amplitude converter (TAC) and a Multichannel Analyzer (MCA), which measured the time delay between the laser pulse and the detected photons.

excitation at the wavelength of $532 \mathrm{~nm}$ is provided by second harmonic generation of a Nd:VAN mode-locked laser (Time-Bandwith GE-100) emitting 6.4 ps pulses at a repetition rate of $113 \mathrm{MHz}$, properly attenuated by a neutral density (ND) filter to achieve the single photon regime. The time resolution of the entire system (limited by the SPAD temporal response) was estimated with a direct measurement of the train of pulses without any sample between the source and detector. The actual laser pulse measured with this configuration exhibits an asymmetric shape with a FWHM of about 30 ps.

\section{Results and discussion}

The spectral speckle intensity correlation technique was used for studying the photon transport properties of Teflon, and in particular for recovering its transport mean free path $\ell^{*}$ and absorption length $s_{a}$. Different slabs of Teflon ranging between $6.25<L<15.15 \mathrm{~mm}$, were characterized in the transmission geometry using the setup described in Fig. 1 and the procedure described in Section 3.1.

The random media temporal response $p(t)$ recovered for each thickness $L$ was converted into the path length distribution $P(s, L)$ after change of variable $s=v t$, where $v$ is the speed of light in Teflon $v=c / n \approx 0.74 c$ ( $c$ being the speed of light in vacuum) [23]. The so reconstructed path lengths distributions from our samples are shown in Fig. 6 as symbols and compared with the theoretical curves predicted according to Eq. (19). The data fitting, which depends on only two parameters appearing in Eq. (19), i.e. $\ell^{*}$ and $s_{a}$, was carried out in two steps, as suggested in $[8,9]$. First of all, since the long path tail of the distribution can be described by a single exponential decay $P(s) \propto \exp \left(-s / s_{c}\right)$ with the rate $1 / s_{c}$ given by

$$
\frac{1}{s_{c}}=\frac{1}{s_{a}}+\frac{\pi^{2}}{L^{2}} \frac{\ell^{*}}{3}
$$

we carried out a single exponential decay fit of the data at the large asymptotic path lengths, and recovered $s_{c}$. Then, the behavior of $1 / s_{c}$ as a function of $\pi^{2} / L^{2}$ for different values of $L$ (see inset, open squares), was fitted according to Eq. (22) and from the fit (solid straight line) we recovered $\ell^{*}=247 \pm 8 \mu \mathrm{m}$ and $s_{a}=685 \pm 25 \mathrm{~mm}$. 


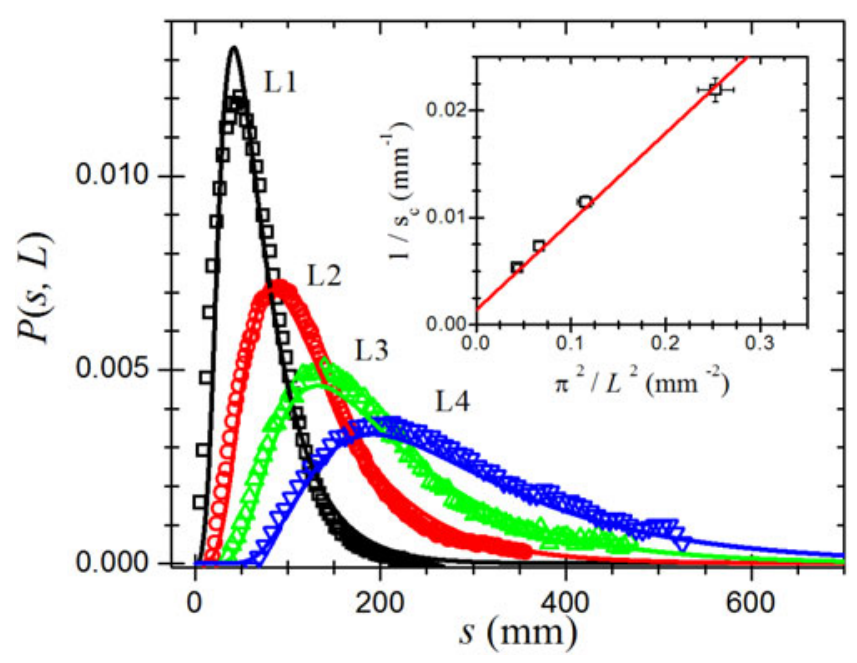

Fig. 6. $P(s, L)$ reconstructed from speckle SSIC technique for Teflon slabs of different thickness (symbols): $L 1=6.25 \mathrm{~mm}, L 2=9.25 \mathrm{~mm}, L 3=12.25 \mathrm{~mm}$ and $L 4=15.15 \mathrm{~mm}$. Lines are fits to $P(s, L)$ with Eq. (19), where the fitting parameter is $\ell^{*}$. Inset: decay rates, $1 / s_{c}$, of the long paths tails of $P(s, L)$ vs. $\pi^{2} / L^{2}$. The line is the best fit of the data to Eq. (22), where the intercept provides an estimate of $s_{a}$ and the slope is related to $\ell^{*}$.

To check the reliability of this fitting procedure, we fitted also all the data with the entire path length distribution given by Eq. (19), by fixing $s_{a}=685 \mathrm{~mm}$ and letting $\ell^{*}$ as a free parameter. The results are shown as solid lines in the main frame of Fig. 6 and the values of $\ell^{*}$ obtained in this way lead to a mean value $\left\langle\ell^{*}\right\rangle=250 \pm 10 \mu \mathrm{m}$, which is in fairly good agreement with the value obtained from the analysis of the asymptotic decay rate. Moreover, our estimates of the transport mean free path and absorption length are consistent with previous results reported in literature [24].

To complete our analysis, we compared the spectral intensity correlation data with the ones obtained on the same samples from the time of flight technique by using the setup described in Section 3.2. Here, we also converted time- into path length-domain by using $v=c / n \approx 0.74 c$ and recovered the path length distributions $P(s, L)$, which are shown in Fig. 7 as symbols. As done for the SSIC analysis, we first fitted the long path tails of the distributions with single exponentials and recover, for every thickness $L$, the decay rate $1 / s_{c}$ (see inset, open squares). Then the behavior of $1 / s_{c}$ was linearly fitted as a function of $\pi^{2} / L^{2}$ via Eq. (22) and from the fit (straight line in the inset) we recovered $\ell^{*}=180 \pm 8 \mu \mathrm{m}$ and $s_{a}=461 \pm 20 \mathrm{~mm}$. At this point, as done for the SSIC data, we could have fitted all the data with the entire paths length distribution given by Eq. (19), by fixing $s_{a}=461 \mathrm{~mm}$ and letting $\ell^{*}$ to be the only fitting parameter. However, differently from the correlation case, we had to take into account the finite resolution of the method and, consequently, the actual fitting function was not given by Eq. (19), but by its convolution with the response of the incident laser pulse. The results of these fittings, shown as solid lines in the main frame of Fig. 7 gave a mean value of the transport mean free path $\left\langle\ell^{*}\right\rangle=181 \pm 6 \mu \mathrm{m}$, in excellent agreement with the value obtained from the decay rate analysis.

Summarizing, a direct comparison between the measurements performed on Teflon slabs of different thicknesses with the spectral intensity correlation and time of flight experiments indicate that the two techniques produce equivalent results. In particular, the recovered path length distributions are fairly similar and, in both cases, well described by the diffusion approximation for light propagation in turbid media, Eq. (19), 


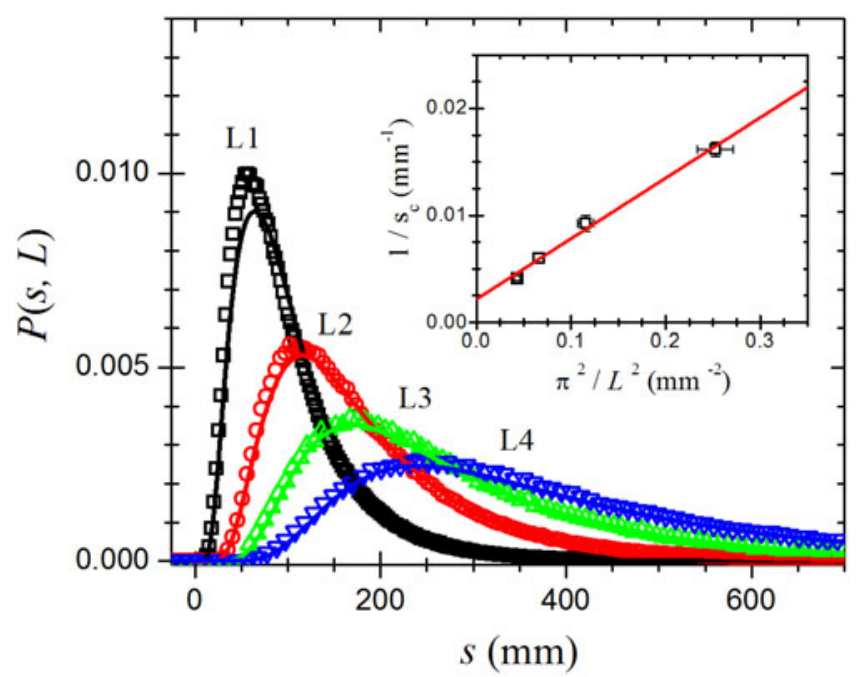

Fig. 7. $P(s, L)$ measured with the photon time of flight technique for the same Teflon slabs of Fig. 6 with thicknesses (symbols): $L 1=6.25 \mathrm{~mm}, L 2=9.25 \mathrm{~mm}, L 3=12.25 \mathrm{~mm}$ and $L 4=15.15 \mathrm{~mm}$. Lines are fits to $P(s, L)$ with Eq. (19) convoluted with the incident laser pulse, where the fitting parameter is $\ell^{*}$. Inset: decay rates, $1 / s_{c}$, of the long paths tails of $P(s, L)$ vs. $\pi^{2} / L^{2}$. The line is the best fit of the data to Eq. (22), where the intercept provides an estimate of $s_{a}$ and the slope is related to $\ell^{*}$.

as illustrated by solid lines in Fig. 6 and 7. However, since the working wavelengths of the two techniques are not the same $\left(\lambda_{\text {freq }}=784.5 \mathrm{~nm}\right.$ and $\left.\lambda_{\text {pulsed }}=532 \mathrm{~nm}\right)$ we obtain different values for the parameters $\ell^{*}$ and $s_{a}$ in each experiment. Interestingly, we notice that the ratio between the values of the two $\ell^{*}$ obtained with the two methods, $\ell_{\text {freq }}^{*} / \ell_{\text {pulsed }}^{*} \approx 1.38$, is close to the ratio of the two wavelengths $\lambda_{\text {freq }} / \lambda_{\text {pulsed }} \approx 1.47$. This difference can be understood by the following simple argument: if the composition and refractive index remains unchanged, as it is the case here [23], the dimensionless scattering strength $\left(k \ell^{*}\right)^{-1}$ can be written as a unique function of the scattering parameter $x=k R$. Here $R$ is the typical radius of a dielectric inhomogeneity. Note that the scattering anisotropy parameter for Teflon is typically $g \approx 0.9$ which means the scattering is deep in the Mie regime. As a direct consequence $\left(k \ell^{*}\right)^{-1}$ only decreases weakly as a function of $x$, in particular if Mie resonances are smeared out by size and shape polydispersity of the dielectric inhomogeneities [25]. From this we can derive an upper-bound estimate $\lambda_{\text {freq }} / \lambda_{\text {pulsed }}=784.5 / 532 \approx 1.47$ in good agreement with our experimental results.

\section{Conclusions}

In this work we have shown that the spectral speckle intensity correlation (SSIC) technique can be profitably used for investigating the photon transport properties of random turbid media. We have developed a simple setup for characterizing samples in the transmission geometry, similar to the one proposed by Webster et al. [12,13], but capable of scanning larger ranges of sample thicknesses and frequencies.

We have used this setup for studying the transport properties of Teflon over a large range of sample thicknesses, $6.25 \mathrm{~mm}<L<15.25 \mathrm{~mm}$, at the working central wavelength of $\lambda=784.5 \mathrm{~nm}$. The photon path length distributions recovered for each $L$ were accurately fitted with theoretical curves predicted according to the photon 
diffusion approximation theory, which allowed us to estimate the Teflon transport mean free path $\ell^{*} \approx 250 \mu \mathrm{m}$ and the absorption length $s_{a} \approx 685 \mathrm{~mm}$. These figures were consistent with previous results available in literature, but obtained with different methods [24], and provided an a posteriori confirmation that the weak scattering $\left(\ell^{*}>\lambda\right)$ and diffusive regime $\left(\ell^{*} \ll s_{a}, \ell^{*} \ll L\right)$ requirements for the applicability of the photon diffusion approximation were both fulfilled by our samples.

We have also tested the accuracy and reliability of the technique by comparing it with complementary measurements carried out with a standard pulsed laser time of flight technique (carried out at $\lambda=532 \mathrm{~nm}$ ) which provides a direct measure of the temporal response of the scattering medium by using the powerful method of TCSPC (time correlated single photon counting), nowadays widely used in time resolved bioimaging. These results were consistent with the ones obtained with the SSIC technique, provided that the finite resolution of the time of flight method and the dependence of $\ell^{*}$ on the different working wavelengths were properly taken into account.

In conclusion, we think that the approach of the SSIC technique can strongly benefit applications in the fields of soft- and bio-materials analysis, as well as diffuse light imaging techniques.

The experiment has been performed in the context of STELLA, the School for Training in Experiments with Lasers and Laser Applications, hold at the Insubria University in Como from June 20 to July 8, 2011 (see www.stella-school.eu). The Authors wish to acknowledge CARIPLO, UNIVERCOMO and Banca del Monte di Lombardia Foundations for having financed the project. The Authors like also to acknowledge the STELLA-school students V. D'Ambrosio, M. Molteni, M.C. Pigazzini, P. Ragazzi, M. Shalaby for their contribution in developing the experimental apparatus, in performing the measurements and in implementing the analysis software, and Prof. P. Di Trapani for fund raising and project coordination. The Authors thank also M. Bondani and L. Nardo for the time of flight measurements on Teflon samples. FS acknowledges financial support by the Swiss National Science Foundation (projects No. 200020-126772, 200021-132736 and 310030-110005).

\section{References}

1. M.C.W. van Rossum, T.M. Nieuwenhuizen, Rev. Mod. Phys. 71, 313 (1999)

2. F. Scheffold, R. Cerbino, Curr. Opin. Colloid Interface Sci. 12, 547 (2007)

3. J.W. Goodman, in Laser Speckle and Related Phenomena, 2nd edn., edited by J.C. Dainty (Springer-Verlag, Berlin, 1984)

4. J.W. Goodman, Statistical Optics (J. Wiley \& Sons, New York, 1985)

5. S.E. Skipetrov, J. Peuser, R. Cerbino, P. Zakharov, B. Weber, F. Scheffold, Opt. Express 18, 14519 (2010)

6. A.Z. Genack, Phys. Rev. Lett. 58, 2043 (1987)

7. M.P. van Albada, J.F. de Boer, A. Lagendijk, Phys. Rev. Lett. 64, 2787 (1990)

8. A.Z. Genack, J.M. Drake, Europhys. Lett. 11, 331 (1990)

9. A.Z. Genack., in Scattering and Localization of Classical Waves in Random Media, edited by P. Sheng (World Scientific, Singapore, 1990), p. 207

10. J.D. McKinney, M.A. Webster, K.J. Webb, A.M. Weiner, Opt. Lett. 25, 4 (2000)

11. E.I. Blount, J.R. Klauder, J. Appl. Phys. 40, 2874 (1969)

12. M.A. Webster, K.J. Webb, A.M. Weiner, Phys. Rev. Lett. 88, 033901 (2002)

13. M.A. Webster, K.J. Webb, A.M. Weiner, J. Opt. Soc. Am. A 20, 2057 (2003)

14. F. Scheffold, W. Härtl, G. Maret, E. Matijevic, Phys. Rev. B 56, 10942 (1997)

15. I.S. Reed., IRE Trans. Inform. Theory IT-8, 194 (1962)

16. A.W. Lohmann, B. Wirnitzer, Proc. IEEE 72, 889 (1984)

17. H. Bartelt, A.W. Lohmann, B. Wirnitzer, Appl. Opt. 23, 3121 (1984) 
18. A. Ishimaru, Wave Propagation and Scattering in Random Media (Academic Press, New York, 1978)

19. R.C. Haskell, L.O. Svaasand, T.T. Tsay, et al., J. Opt. Soc. Am. A 11, 2727 (1994)

20. M.S. Patterson, B. Chance, B.C. Wilson, Appl. Opt. 28, 2331 (1989)

21. D.D. Duncan, S.J. Kirkpatrick, R.K. Wang, J. Opt. Soc. Am. A 25, 9 (2008)

22. A. Andreoni, M. Bondani, L. Nardo, Mol. Cell. Probes 28, 119 (2009)

23. M.K. Yang, R.H. French, E.W. Tokarsky, J. Micro/Nanolith. MEMS MOEMS 7, 033010 (2008)

24. A.C. Völker, P. Zakharov, B. Weber, F. Buck, F. Scheffold, Optics Express 13, 9782 (2005)

25. M. Reufer, L.F. Rojas-Ochoa, S. Eiden, J.J. Sáenz, F. Scheffold, Appl. Phys. Lett. 91, $171904(2007)$ 